\title{
Some General Remarks on Histological Diagnosis
}

Makek M: Clinical Pathology of Fibro-Osteo-Cemental Lesions in the Cranio-Facial and Jaw Bones. Basel, Karger, 1983, pp 10-13

Morphologically speaking, the conditions known as 'fibro-osteo-cemental lesions' are generally characterized by the fact that they contain few components. Stroma comprising connective tissue is always present, though the density ofits cell content may vary, and it is this stroma which produces the hard min- eralized component of the lesion. The tissue produced can either be woven bone, which may or may not contain osteoblasts, or la- mellar bone tissue, or even on occasion pecu- liarly layered, spherical, darkly colared struc- tures which are referred to as 'cementicles'. The more recent literature published on the subject of 'fibroosteo-cemental lesions' has attempted to isolate the various entities covered by the term and to differentiate them from each other. The presence or absence of cementum-like histological structures has of- ten been regarded as a distinguishing charac- teristic in this connection. It has also been maintained, in a study by Reed [1963], that a lesion can be correctly diagnosed as fibrous dysplasia only ifit contains no lamellar bone. In the past, the presence of lamellar bone in lesions which had been diagnosed as fibrous dysplasia has caused a considerable amount ofconfusion. An earlier study by Reed [1959] rcpresents what one might describe as the 
'purist' point of view. 1t holds that polyos- totic fibrous dysplasia is caused by matura- tion of a woven bone coming to a standstill when it is in its metaplastic phase, on which point it concurs with the earlier work by 
Lichtenstein [1938]. The view that it was pos- sible for fibroblasts tobring about metaplasia of the bone was partly based on the histo- chemical findings of Changus [1957] and Jeflree and Price [1965]. Many authors have observed high alkaline phosphatase activity in the fibroblasts found in fibrous dysplasia lesions, and it is certainly an indication that these stroma cells do have osteob1astic poten- tial. Nevertheless, Pep/er [1956] did describe cases of ossifying fibroma which had similar histochemical attributes. The purists hold that the presence of lamellar bone or periph- eral osteoblastic structures utterly rules out any diagnosis offibrous dysplasia. One study whose findings are somewhat at variance with this viewisthat by laffe [1953]. In it he states that when fibrous dysplasia occurs in the skull, it ossifies faster than in other bones.

Waldran [1970] and Dahlgren et al. [1969] regard the presence of trabeculae of both woven and lamellar bone and peripheral osteoblastic structures as possible in cases of fibrous dysplasia. A study by Eversole et al. [1972] covering 75 cases of monostotic fi- brous dysplasia in other bones and 34 cases of cranio-facial fibrous dysp1asia shows that in $39 \%$ of the cases in the first group and $37 \%$ of those in the second, lamellar bone occurs. When lamellar bone is present, care should be taken to distinguish it from periph- eral, reactive bone formations.

Spheroid structures, similar in appearance 
to cementicles and grouped tagether in layers, often predominate in all or at least part of the tissue affected by fibro-osseous lesions. When they do occur, it is difficult to determine precisely what effect they should have on the diagnosis returned. A number of authors [Cahn. 1951; Zimmerman et al., 1958; Hamner et al., 1968; Small and Goodman, 1973; Tomeo, 1978] found ovoid calci- fications of this type to be present in many cases of ossifying fibroma of the jaw. In view ofthe similarity between these structures and genuine cementicles, these authors describe the conditions in which they are present as 'cementifying fibroma' or 'cemento-ossifying fibroma'. Indeed, Hamner et al. [1968] di- vide fibroma up into four separate sub- groups, which they describe as cementoid, osteoid, mixed (i.e. cemento-osteoid) and fi- brous respectively. The criteria used to distinguish between these four subgroups are (1) the presence or absence of calcification; (2) the chromatic characteristics of these struc- tures when stained with hematoxylin and eo- sin; (3) the distance between the dark and light lines of parallel birefringence presented by the trabeculae of mineralized tissue when viewed microscopically under polarized light. In 
their study, Waldran and Giansanti [1973 b] do not appear to contest these dis- tinctions, though they are noticeab1y cautious in their approach towards them. In their view, it would be more appropriate to classify cementifying and ossifying fibroma as sepa- rate and distinct phases ofthe same patholog- ical process.

Eversole et al. [1972] found that these cementicle-like 1ayered structures occurred in $15-20 \%$ of the cases of cranio-facial fibrous dysplasia covered by their study. A number of other authors found these cementicle-like structures in fibro-osseous lesions occurring 
in non-cranio-facial bones. Van Horn et al. [1963], for example, cite ten cases of non- cranio-facial fibrous dysplasia in which parti- cles of cementum were present, and Fried-man and Goldman [1969] mention two cases of a lesion they term 'cementoma', one oc- curring in the humerus and the other in the femur. Volume 19 of the WHO classification [Shanmugaratnam, 1978] contains a slide showing cementifying fibroma occurring in the paranasal cavities.

Langdon et al. [1976] proposed that the distinction between ossifying and cementify- ing lesions be dropped since it is not possible, on the basis of a histological examination, to differentiate between bone and cementum with absolute certainty and since cementumlike calcifications have been shown to occur in fibro-osseous lesions whatever their loca- tion. These authors also put forward the view that ossifying fibroma is not the only patho- logical entity ofits kind, but rather just one of a whole range of fibro-osseous lesions. In their view, this range of fibro-osseous lesions should also include fibrous dysplasia. Chro- matic, histochemical and optical criteria have all been applied in the numerous at- tempts which have been made to differen- tiate between osteoid and cementum. So far, no method has yet been evolved which can produce absolutely satisfactory and defini- tive results. Hamner et al. [1968], for exam- ple, state that genuine cementum particles are characterized by their shape, which is similar to a droplet or a sphere, and by the fact that 
their matrices have relatively low cell density. They go on to remark that, although coarse cells or cementoblasts can be observed in the peripheral area of the fibro- osseous lesions they describe as 'cementify- ing fibroma' and 'cemento-ossifying fibro- ma', they are neither as prominent nor as 12 Clinical Pathology of Fibro-Osteo-Cemental Lesions

Table I. Histological typing of odontogenic tumors, jaw cysts, and allied lesions [Pindborg and Kramer, 1971]

I Neoplasms and other tumors related to the odontogenic apparatus

\section{A Benign}

Amelob1astoma

2 Ca1cifying epithelial odontogenic tumor

3 Ameloblastic fibroma

4 Adenomatoid odontogenic tumor

(adenoameloblastoma)

S Calcifying odontogenic cyst

6 Dentinoma

7 Ameloblastic fibro-odontoma

8 Odontoameloblastoma

9 Complex odontoma

10 Compound odontonia

II Fibroma (odontogenic fibroma)

12 Myxoma (myxofibroma)

13 Cementomas

a) Benign cementoblastoma (true cementoma)

b) Cementifying fibroma 
c) Periapical cemental dysplasia (periapical fibrous dysplasia)

d) Gigantiform cementoma (familial multiple cementomas)

14 Melanotic neuro-ectodermal tumor of infancy

(melanotic prognoma, melano-ameloblastoma)

\section{B Malignant}

Odontogenic carcinomas

a) Malignant ameloblastoma

b) Primary intraosseous carcinoma

c) Other carcinomas arising from odontogenic epithelium, including those arising from odontogenic cysts

2 Odontogenic sarcomas

a) Ameloblastic fibrosarcoma (ameloblastic sarcoma)

b) Ameloblastic odontosarcoma 
II Neoplasms and other tumors related to hone

\section{A Osteogenic neoplasms}

I Ossifying fibroma (fibro-osteoma)

B Nonneoplastic bone lesions

Fibrous dysplasia

2 Cherubism

3 Central giant cell granuloma

(giant cell reparative granuloma)

4 Aneurysma!bone cyst

S Simple bone cyst

(traumatic, hemorrhagic bone cyst)

111 Epithelial cysts

\section{A Developmental}

Odontogenic

a) Primordial cyst (keratocyst)

b) Gingival cyst c) Eruption cyst

d) Dentigerous (follicular) cyst

2 Nonodontogenic

a) Nasopalatine duct (incisive canal) cyst b) Globulo-maxillary cyst

c) Naso-labial (naso-a1veolar) cyst

B Inflammatory

Radicular cyst

IV Unclassified lesions

Some General Remarks on Histological Diagnosis 
numerous as the osteoblasts which occur in the peripheral areas of dassie fibrous dyspla- sia lesions. Giansanti [1970] gives details of other characteristics of cementum which he regards as distinctive and which have appar- ently enabled him to distinguish successfully between cementum and osteoid. He found, for example, that cementum tended to be magenta-colored and was highly basophilic when subjected to hematoxylin and eosin stain tests, whereas osteoid was markedly acidophilic. He also states that cerneoturn has a characteristically regular structure of collagenaus fiber bundles which can be de-tected microscopically under polarized light. In the course of his investigations with a polarization microscope, he observed that the dark and light lines of parallel birefrin- gence presented by the trabeculae were fine in cases where cementum was present, whereas they were noticeably coarser and more widely spaced when osteoid was involved. These observations raised a number of interesting questions which were considered in detail in the study by Eversole et al. [1972].

If the calcified material contained in a cranio-facial lesion is diagnosed as cemen- tum, one is then faced with the problematic task of determining precisely what type of ce- mentallesion is involved. Periapical cemen- tal dysplasia and cementifying fibroma are the two cemental lesions which are the most difficult to distinguish from 
fibrous dysplasia and ossifying fibroma. In many cases it would seem impossible to differentiate between ce- mentifying and ossifying lesions on the basis of histological evidence alone. Makek [1977] published a study which attempted to distin- 
guish definitively between fibrous dysplasia and ossifying fibroma. As a first step the total surface area of each of the lesions considered was measured and expressed in terms of square millimeters. In each case this total surface area was then compared to the area cov- ered by newly formed bone and spindle-eeil stroma. 1t emerged that in cases of fibrous dysplasia the proportion of the total surface area of the lesion occupied by newly formed bone tissue and spindle-eeil stroma remained constant from case to case, whereas in cases of ossifying fibroma it varied considerably. As a criterion for differential diagnosis, this rela- tionship between the surface area ofthe lesion covered by newly formed bone tissue and spindie cells and the total surface area of the lesion provided satisfactory results. However, it should be pointed out that this study con- sidered lesions occurring in the ribs only, and that when the same technique was used to dif- ferentiale between the same types of lesion occurring in the jaw-bones, the results ob- tained were inconclusive.

The diagnosis of osteogenic and odonto-

genic lesions of the jawbones was brought a great step forward when Findborg and Kram-er's [1971] WHO classification was published (table I). This was the first comprehensive description of these lesions and the first to recommend terminology for international use. As such, it has undoubtedly fulfiiled its authors' stated aim of facilitating and im- proving communication among cancer work- ers. As the authors point out, it reflects the state 
ofknowledge at the time (1971) and will almost certainly have to undergo modifica- tions as experience accumulates. 